\title{
Congenital Absence of pericardium
}

\author{
Sharma D, Baidya SG, Prajapati D, Lamsal K, Kayastha B, Pant S, Ghimire RK \\ Shahid Ganga Lal Nationa Heart Centre, Bansbari, Kathmandu
}

A 30- year-old physically active male presented to us for evaluation of left sided chest pain which was non-exertional in nature. He described it as being of pricking type and non-radiating. His past history was not significant. Physical examination revealed a non-palpable apical impulse with no other abnormal findings.

A chest $\mathrm{X}$-ray ( $\mathrm{P}$-A view) revealed levoposition of heart, loss of the right heart border (hidden by spine) and prominence of pulmonary artery segments(Fig 1).A "tongue" of lung interposing between aorta and pulmonary artery was not evident in chest $x$-ray in this case.

ECG was taken in the supine position with precordial leads placed in the standard position , which revealed right axis deviation, right bundle branch block(RBBB) pattern and poor $\mathrm{R}$ wave progression(Fig2).

Transthoracic echocardiography revealed unusual windows with marked lateral displacement of apical windows. Lateral placement of the transducer was also necessary for parasternal axis views. Right atrium and right ventricles were mildly dilated. Echocardiography excluded other structural heart diseases (Fig 3 and Fig 4).

As the above findings were suggestive of the diagnosis of congenital absence of pericardium, further investigations including CT chest and cardiac MRI were done to confirm the diagnosis. CT thorax(Fig 5), also suggested absence of pericardium.

Axial T1 weighted cardiac MRI revealed markedly rotated axis of the the heart with mildy dilatation of ventricles. Lung was seen insinuating between aorta and pulmonary artery. Pericardium was absent(Fig 6). Thus, the diagnosis of total absence of pericardium was confirmed.

Patient was advised to continue activities without limitation and was not referred for any kind of intervention.

Fig 1
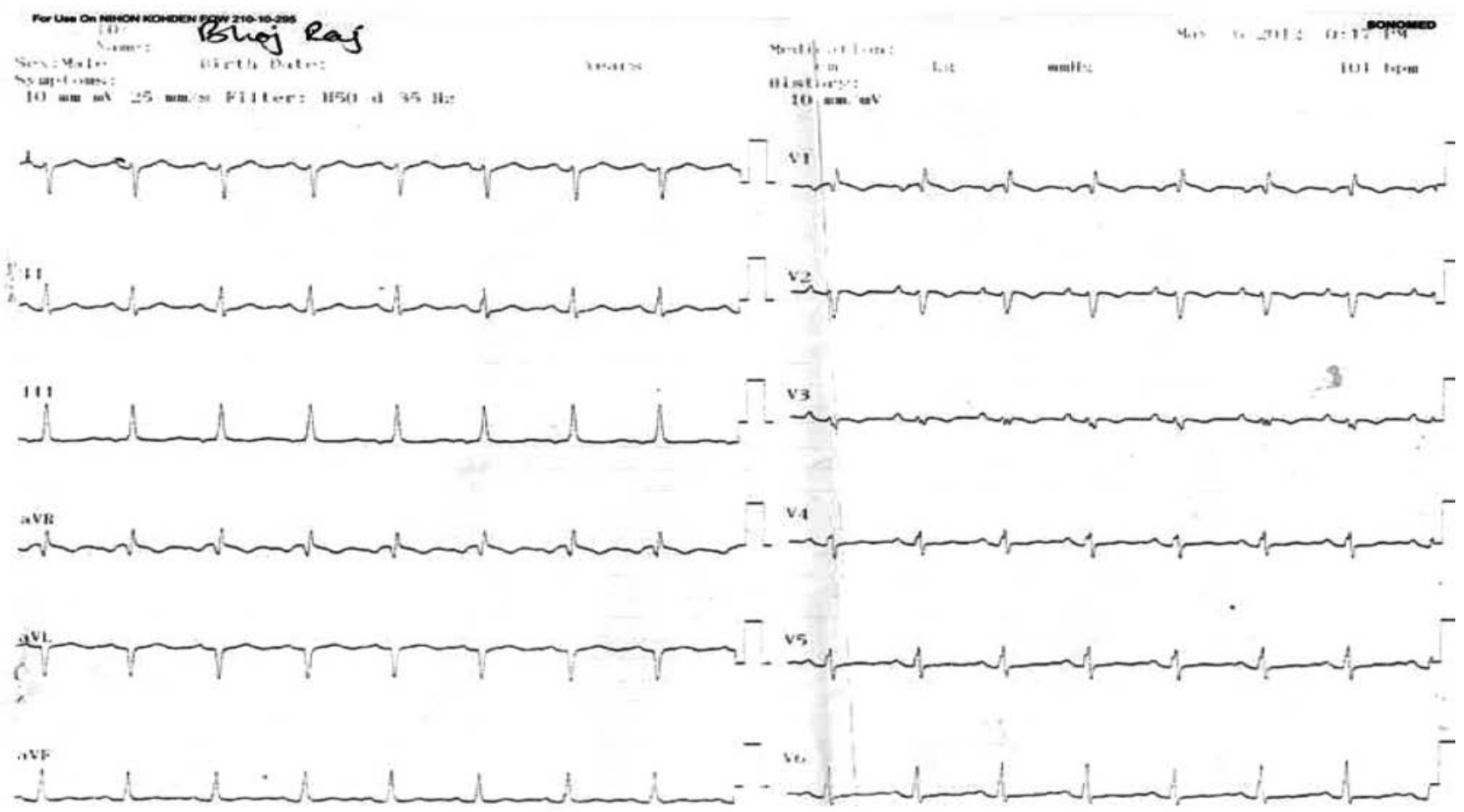
Fig 2.
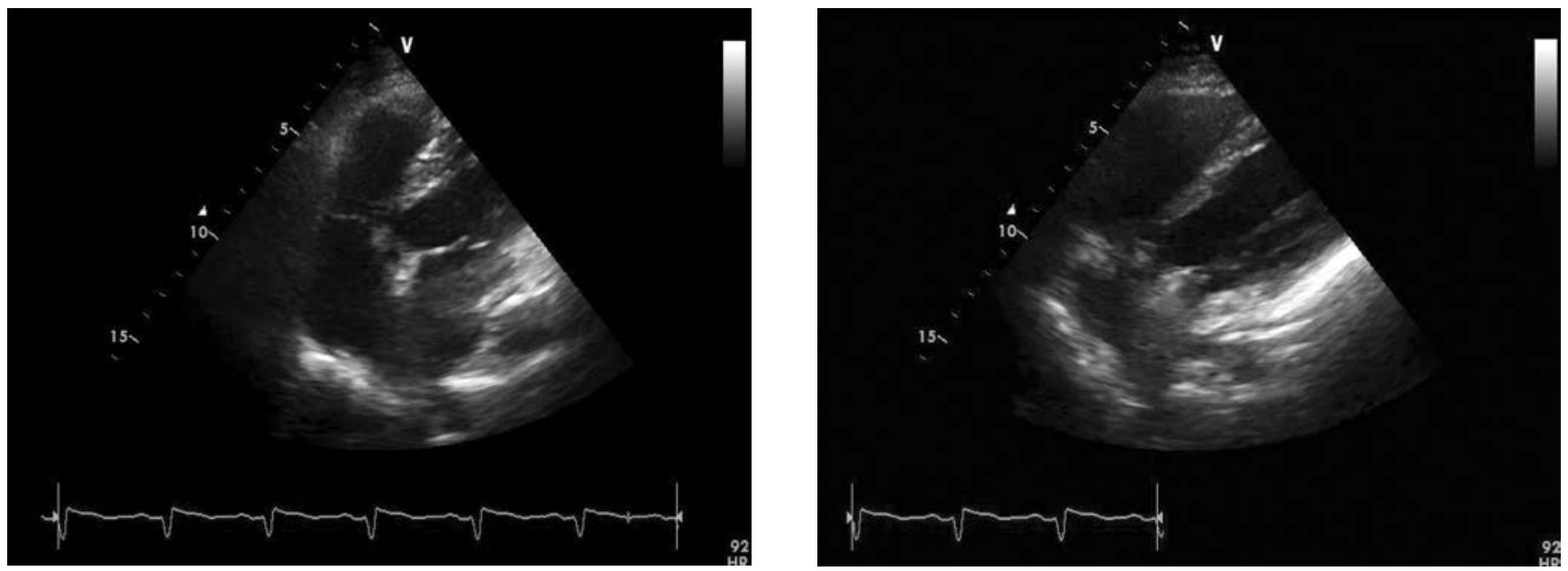

Fig 3.

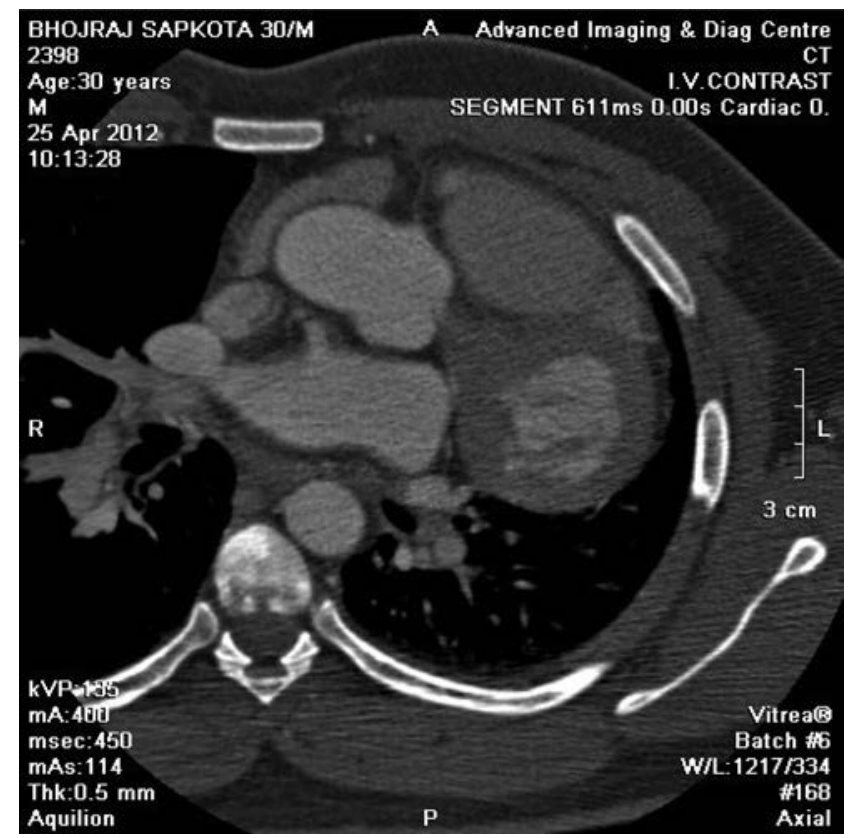

Fig 4

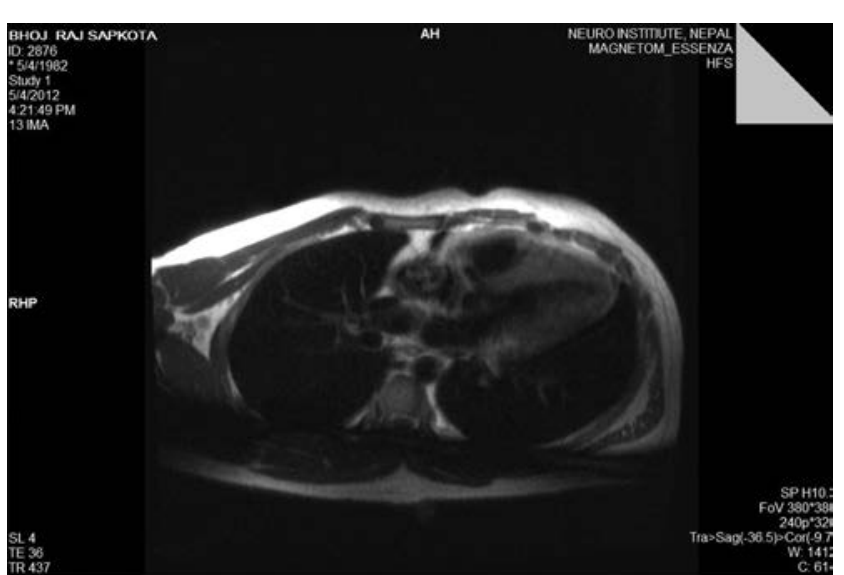

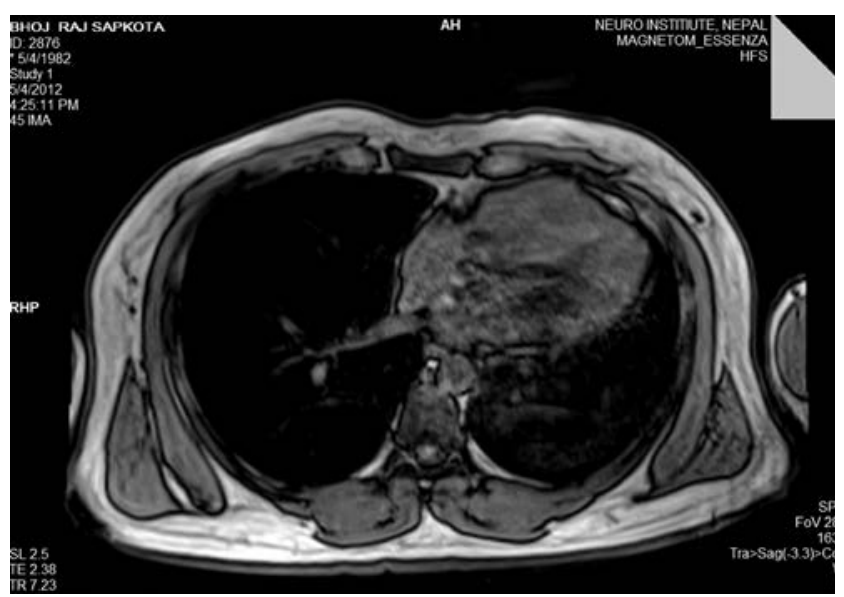




\section{Discussion}

Isolated congenital absence of pericardium includes a range of congenital pericardial defect from a small foramen in the pericardium to a complete absence of the entire pericardium ${ }^{1}$.The clinical presentation is non-specific. In most reported cases, the problem was detected incidentally in asymptomatic individuals. However, some subjects may present with symptoms of atypical chest pain, dyspnoea and palpitations. Physical examination may reveal a laterally displaced apex or absent apical impulse and systolic murmurs and clicks of undetermined origin. ${ }^{2}$ Patient may also present with complications clearly related to the defect, most common one being herniation and incarceration of the myocardium, predominantly left atrial appendage 4 . Ventricular herniation has been also described ${ }^{5}$.Other complications include torsion of great vessels secondary to increased cardiac mobility, coronary artery compression by the edge of pericardial defect. All these complications have been associated with presentations varying from chest pain to infarction, syncope, tricuspid regurgitation and sudden death ${ }^{6}$.Therapeutic options are mainly based on small retrospective series. It is recommended that patients presenting with complications should be treated with surgery. For those without complications various strategies have been proposed. Total or large defects without complications do not need surgery as it was in our case. Small or moderate size defects can be considered for surgery. Surgical options include both pericardiotomy or pericardioplasty. The former enlarges the defect to reduce the risk of incarceration while the later attemps to achieve the same goal by direct closure or closure of the defect by a synthetic material.

\section{Reference}

1. Nasser WK. Congenital diseases of the pericardium. Cardiovasc Clin 1976;7:271-286

2. Staeve Brulotte, Louis Roy, Eric Larose. Congenital absence of the pericardium presenting as acute myocardial necrosis. Can J cardiol 2007 Sep:23(11):909-912

3. Subha V Raman, Curt J Daniels, Steven E Katz et al. Congenital Absence of the Pericardium.Circulation, 2001;104;1447-1448

4. Isolated congenital absence of the pericardium: clinical presentation, diagnosis and management. Michael A Gatzoulis, Mare-David Munk, Glen S Van Ardell et al. Ann Thorac Surg 2000;69:1209-1215
5. Saito R, Hutta F. Congenital pericardial defect associated with cardiac incarceration: case report. Am Heart J.1980;100:86670

6. Jones JW, McManus BM. Fatal cardiac strangulation by congenital partial pericardial defect. Am Heart J.1984;107: 183-5 